\title{
SharedSpace Based Service Discovery Mechanism and Its Implementation for Ubiquitous Environments
}

\author{
Sangdo Park, Junhyeong Kim, and Paul Barom Jeon* \\ Communication and Networking Lab., \\ Samsung Advanced Institute of Technology \\ Nongseo Giheung Yongin Gyeonggi 446-712, Korea \\ \{sdpark, skykimjh, paul.barom.jeon\}@samsung.com \\ http://www.sait.samsung.com
}

\begin{abstract}
We propose a new service discovery method based on SharedSpace concept. SharedSpace is a virtual community space for service registration and sharing, which is similar to a chat room in a chat system. Any usen 11 can freely create a SharedSpace and register his/her services. Others can join the created SharedSpace as members and register their services. All registered services can be shared by the SharedSpace members as if they are in a single network. Detailed mechanism is designed and implemented with Obje middleware in order to validate our scheme.
\end{abstract}

\section{Introduction}

Advances in networking and computing technology have progressed to a point of real ubiquitous computing environments. Consequently, researchers come to show interest in complex usage scenarios as well as ad-hoc connectivity issues [1]. Various connectivity technologies have been discussed so far, but are not able to cover all scenarios satisfactorily yet even though most of the issues can be resolved [2].

Early ubiquitous network's usage scenario mainly focused on connectivity issues among nodes that compose ubiquitous network. Network-enabled devices such as camera phone, MP3 player, Portable Media Player (PMP), etc., are increasing explosively and various solutions are presented also in reply. On the other hand, a tendency to separate contents and devices occurs because it causes several inconvenience to carry hundreds of GB multimedia contents. This means that ubiquitous computing environments require connectivity among nodes over the network boundary as well as inside the network [3].

Before connecting nodes, one should achieve service/component discovery first. Conventional service discovery methods can be classified into two categories: multicast and directory server based methods. However, multicast based

\footnotetext{
* Corresponding author.

${ }^{1}$ In this paper, user is defined as a person who is connected to a server. Participant is an user joining a SharedSpace and clinet is a program run by the user.
} 
service discovery can't find services beyond network boundary. Note that listing up available services instead of querying a specified service, will give users more flexibility to compose a desirable service under ad hoc ubiquitous environments.

Therefore, in this paper, a new service discovery method that can be utilized in ad hoc ubiquitous network environments is proposed. The proposed method delegates mDNS 4 to discover services on the local network. Whereas, for service discovery beyond network boundary, a new concept of SharedSpace similar to a chat room in a chat system is suggested.

\section{Service Discovery Using ShardSpace}

SharedSpace is a kind of tiny database(DB) on the public network server(Shared Space Server). It can contain device and service information that are registered by participants authorized to access the shared space. As people open a chat room, any participant can create a SharedSpace. Participants can upload device and service information as people talk to other persons. We can imagine that a participant joins the SharedSpace and sends some information on service and devices available locally. Once a new information is uploaded, this information can be transferred to all participants. As devices or services are not available any more, participants can get rid of the information from the SharedSpace. Similar to general discovery systems, uploading and deleting information are dynamically performed depending on local network environments. Figure 1 shows an example of SharedSpace that contains information about services and devices.

To get information about services, user should connect to the Shared Space Server first using client application. Client application can be run on any network device. As user connects to server, it receives the list of SharedSpaces created before. Every SharedSpace has Title for identification. Authentication and authorization to enter the SharedSpace can be adapted to the user for access control. On entering the SharedSpace, user can get current service information registered before. All participants can upload local service information and participants inside the SharedSpace should be notified about it. For some reason, service may not be available on any moment. So, users should be able to revoke services on the SharedSpace. Revoked services should also be notified to all participants belonged to the same SharedSpace.

Shared Space Server

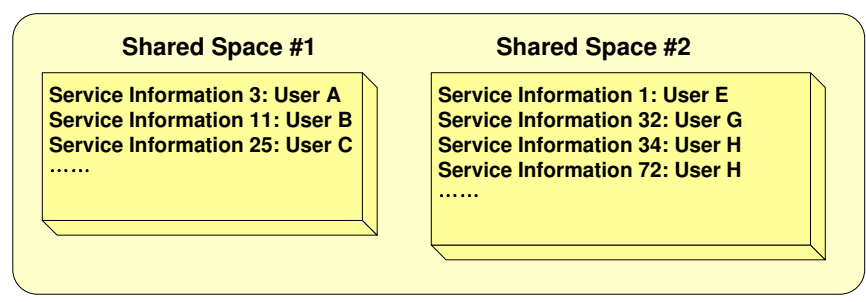

Fig. 1. An example of Shared Space 
If there is no adequate SharedSpace, user can generate a new one with an identification. New identification should be announced to all users connected to server for potential joining. Similarly, SharedSpace can be removed from Shared Space Server if it is not used any more. For proper operation, SharedSpace should be equipped with server and user functionality. Server should be able to create DB for each SharedSpace. Optionally, server can authenticate and authorize users to offer access control. On the other side, user functionality gives user to connect to server and to generate UI for selecting SharedSpace as well as to achieve service discovery on local network utilizing multicast discovery method such as mDNS.

The proposed method has following features: First, it is not restricted to only two participants. Through one SharedSpace, more than two users can upload services to support abundant service pool. Moreover, even though the number of services increase, clients don't need to know each service description a priori. Participant should only select a SharedSpace he/she wants to join. Secondly, user can join more than two SharedSpaces simultaneously. Naturally, a participant can use some services provided separately by other SharedSpace. Although same services are provided by more than two subnetworks, participant can identify each using context information such as subnetwork name, location, etc. Finally, sharing service information only within SharedSpace enables a natural access control.

The mechanism can discover various services inside and outside of the local network without specific service descriptions. However, it should solve how a client could select the proper SharedSpace to participate in among many available SharedSpace. That is users in each subnetwork should be concerned with service directly(user-in-the-loop) and users should agree on the title of the SharedSpace. In other words, the proposed method requires that each subnetwork should have at least one client, for the services to be registered and become accessible. We can assume that user is controlling the client because proposed mechanism targets ubiquitous computing environment. If there is no user in a subnetwork, we can distribute a nominal client to register services on a fixed network to a specific SharedSpace. Also, we assume that the SharedSpace's title acquisition issue can be solved by using side communication channels such as internet messenger, e-mail, voice communication etc.

\section{Practical Implementation}

We utilized Obje [5] middleware to implement the proposed scheme and validate its efficiency and ability. Obje is a peer-to-peer communication middlware over IP network that supports recombinant computing. An implementation example which consisted of two subnetworks was depicted in Figure 2 The subnetwork named My Home in the left contained Webcam device, microphone, TV set, and PC. An user could discover and utilize services by using the client agent immanent in the TV set. The subnetwork named Parent's Home in the right also contained Webcam device, microphone, TV set, and PC. In addition, it had a network-enabled digital picture frame that could display images. The access point (AP) bridges wired and wireless networks together. 


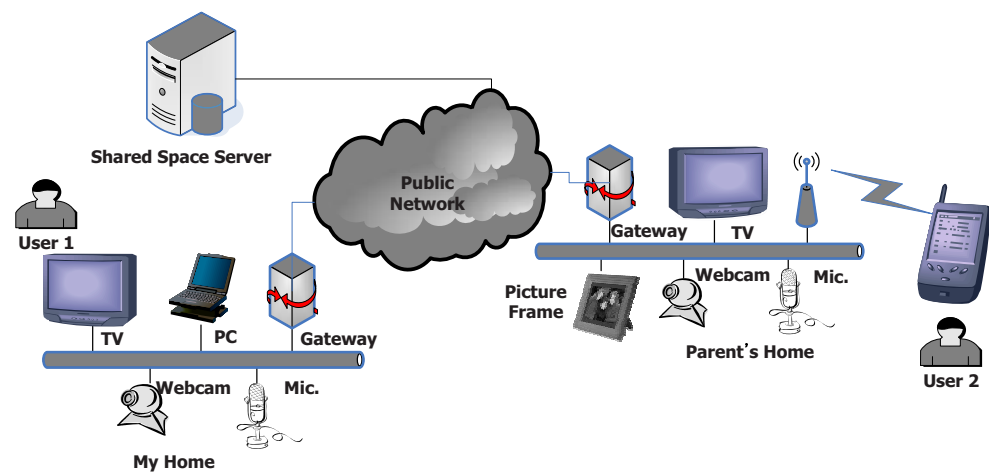

Fig. 2. Network Configuration

Following example scenarios were studied. User 2 with a PDA connected to Parent's Home network forming a new ubiquitous environment. Once the network was formed, the client inside the PDA searched for available services on the local network. The discovered available services were provided to user 2 . At the same time, the client gained access to the Shared Space Server, obtained the list of SharedSpaces and joined the Birthday Plan generated by user 1 on My Home. Then, user 2 got service references of My Home as well as Parent's Home. After gathering the service references of both subnetworks, the client sent them to the Obje middleware. Then, Obje presented specific information of the service. Finally, user 2 connected TV, Webcam, microphone, and speaker of My Home to Webcam, TV, speaker, and microphone of Parent's Home respectively by using the Obje user interface and performed video conference with user 1 .

\begin{tabular}{|l|l|l|l|l|}
\hline Connections & Wander version 2 & \\
\hline Apdate URL \\
\hline A Microphone for sending audio data
\end{tabular}

Fig. 3. User Interface 
This made it possible to utilize large TV screen instead of small PC monitor. Another possible application is to display digital photos saved in My Home's PC to digital picture frame of Parent's Home.

Figure 3 shows the UI for user application. It consists of two panels. The left panel shows the components discovered on the local network and discovered through SharedSpace. The right panel shows the list of SharedSpaces and corresponding control buttons. This figure depicts the aforementioned scenario also. Clients of both User1 and User2 joined the SharedSpace named Birthday Plan. In result, various components of both subnetworks were discovered and displayed. User could connect the components of interest by simply performing drag and drop of the discovered components.

\section{Conclusion}

In this paper, we described a process of performing service discovery mutually for the clients when they belong to different subnetworks. SharedSpace concept was used to hold service information on the server. Clients were to register local services they discovered to the SharedSpace each. All service information of the subnetworks could be shared among clients that joined the SharedSpace. Authorization and authentication on the server enabled ordinary control of the accesses to the services without any additional complex process. We validated out proposed scheme by implementing a video conference application with modified Obje middleware. The proposed method offers a simple guide of how services can be discovered by various application scenarios under ubiquitous computing environments in the future.

\section{References}

1. Stromberg, H., Pirttila, V., Ikonen, V.: Interactive scenarios-building ubiquitous computing concepts in the spirit of participatory design. Personal and Ubiquitous Computing 8(3-4) (2004)

2. Edwards, W.K.: Discovery systems in ubiquitous computing. IEEE Pervasive Computing 5(2), 70-77 (2006)

3. Sivavakeesar, S., Gonzalez, O.F., Pavlou, G.: Service discovery strategies in the ubiquitous communication environments. IEEE Communications Magazine 44(9), 106-113 (2006)

4. Cheshire, S., Krochmal, M.: Multicast DNS. IETF Draft (August 2006) draftcheshire-dnsext-multicastdns-06.txt

5. PARC: Obje: Ineroperability framework (2003), http://www.parc.com/research/projects/obje/Obje_Whitepaper.pdf 